\title{
'Aware of What?' A Formal Model of Awareness Systems That Extends the Focus-Nimbus Model
}

\author{
Georgios Metaxas and Panos Markopoulos \\ Industrial Design, Technical University of Eindhoven, \\ The Netherlands \\ $\{\mathrm{g}$.metaxas, p.markopoulos\} @tue.nl
}

\begin{abstract}
We present a formal-model of awareness-systems founded upon the focus and nimbus model of Benford et al [2] and of Rodden [19]. The model aims to provide a conceptual tool for reasoning about this class of systems. Our model introduces the notions of aspects, attributes and resources in order to expose the communicational aspects of awareness-systems. We show how the model enables reasoning about issues such as deception and plausible deniability, which arguably are crucial for enabling users to protect their privacy and to manage how they present themselves to their social network.
\end{abstract}

Keywords: CSCW, formal models, awareness systems, focus-nimbus, Z.

\section{Introduction}

Awareness systems are communication systems whose purpose is to help connected individuals or groups to maintain awareness of the activities and the situation of each other. In the domain of group-work where awareness systems were first studied, awareness has been defined as "an understanding of activities of others that provides a context for your own activities" [8].

In a more social context, interpersonal awareness can be considered as an understanding of the activities and status of one's social relations, derived from social interactions and communications with them. Casablanca [12] was an early influential project that explored the design space of awareness technology for the domestic environment. Astra [17] studied intentional communication for the extended family and demonstrated that such communication can enhance feelings of connectedness and can prompt rather than replace direct communications. CareNet [6] focused on "Assisted living" by informing professional care-givers as to medication, nutrition, falls, etc., of elderly patients living alone. The Digital-Family-Portrait (DFP) [20] was designed to provide peace of mind to adult children regarding a lone parent living at a distance.

The works cited represent just a tiny fraction of the growing literature on the topic of awareness systems, which expands to an ever increasing variety of physical and social contexts addressing an equally diverse range of user needs. We discern two trends regarding this proliferation of research on awareness:

- The great majority of awareness systems concepts proposed in related literature cluster around some basic themes; some of the most common themes are,

The original version of this chapter was revised: The copyright line was incorrect. This has been corrected. The Erratum to this chapter is available at DOI: 10.1007/978-3-540-92698-6_37

J. Gulliksen et al. (Eds.): EIS 2007, LNCS 4940, pp. 429-446, 2008.

(C) Springer-Verlag Berlin Heidelberg 2008 
communicating to someone that you think about him/her, conveying simple presence information at a particular location, sustained audio video links between places, serendipitous discovery of information about others, supporting flexibility and the conjoint creation of meaning between participants, etc.

- Theoretical discussions motivating the design of such systems gravitate towards the phenomena surrounding the social aspects of using awareness. For example, T.Erickson [9] has introduced the concept of social translucence that encapsulates issues of inter-subjectivity between users of awareness systems. Other issues relate to privacy of people and ways in which they might manage their accessibility to others, (e.g., [13], [3], [14]).

These two trends point to the need for a clear conceptualization of awareness systems that lends some clarity to the description of relevant phenomena. More specifically, such a conceptualization should abstract away from detailed aspects of form and application context, to describe the communication aspects of awareness systems in terms relevant for discussing social interactions between users.

Schmidt [21] discussed the endemic lack of conceptual clarity for the research domain we sketched out above. Noting the contradictory uses of the term awareness, he argued that dichotomies between attention and peripheral awareness, active and passive awareness, explicit and tacit, etc., are misleading. Rather he argued that awareness should be described in reference of activities, practices or phenomena or object that a person is made aware of. In line with this argument, the remainder of the paper presents an abstract model of awareness systems that incorporates related concepts and supports reasoning regarding social aspects of using awareness systems.

\subsection{Related Work}

There have been several attempts to create mathematical abstractions of awareness. Inspired from biology, Bandini et al. [22] proposed the reaction-diffusion metaphor that aimed to make "awareness mechanisms fully visible and accessible to the involved actors for the purpose of adaptability". The model is based on the notions of space, and fields. Space is populated by entities, and it is used to evaluate when entities come in contact and to express how fields propagate in the space. Fields are the means by which awareness information is brought in and propagated in the space, and influences the entities able to perceive it. Mechanisms governing the emission and reception of fields provide the capability of modulating awareness on the side of the emitter as well as of the receiver.

Fuchs et al. [11] suggest an event distribution model for CSCW environments, that can be applied to support shared awareness in systems for the coordination of cooperative work. The model proposes the representation of the environment as a semantic network. Awareness about changes and synchronous activities in the system is supported by the generation and distribution of events in the semantic network.

Benford et al. [2] introduced the notions of Nimbus and Focus in a spatial model of group interaction, in order to address mutual levels of awareness within a virtual environment.

- Focus represents a sub-space within which a person focuses their attention. The more an object is within your focus the more aware you are of it. 
- Nimbus on the other hand represents a sub-space across which a person makes their activity available to others. The more an object is within your nimbus, the more aware it is of you.

Based on these notions Benford et al. define a "measure of awareness" as a functional composition of Focus and Nimbus quantifiers; this measure provides the answer to the question: "In a given room, how aware is entity i of entity $j$ via medium $k$ ?".

Rodden [19] expanded the focus/nimbus model for a wide range of cooperative applications, beyond the boundaries of spatial applications, by using set notation to describe focus, nimbus, and awareness and the operations that can be performed on them.

The focus-nimbus model of Rodden has had several applications since it was introduced. Recently, Cohen et al [10] constructed a first-order logic representation of focus and nimbus enabling the definition of higher level operations for controlling multi-media streams between communicators using higher level operations such as mute, hide, etc. SOGAM (Service Oriented Group Awareness Model) [15], is a recent implementation oriented model, focusing on web services that can support groupawareness. These renditions of Rodden's model are application specific and are not appropriate for supporting a general model of awareness systems and for reasoning for user relevant aspects such as, privacy, translucence, etc.

Privacy and awareness represent flip sides of the same coin. Noting the duality of these needs Boyle and Greenberg [4]applied the concepts of attention, fidelity, and identity in order to define privacy needs in the ubicomp domain. They proposed the following characterizations for privacy needs:

- Solitude: control over one's interpersonal interactions, specifically one's attention for interaction.

- Confidentiality: control over other's access to information about oneself, specifically the fidelity of such accesses.

- Autonomy: control over the observable manifestations of the self, such as action, appearance, impression, and identity.

Boyle and Greenberg go on to project their tripartite conception of privacy on Rodden's focus/nimbus model for awareness. Foci correspond roughly to attention so solitude can be thought of as focus regulation. Nimbi correspond to embodiments and socially constructed personas and to one's relationships with information and artifacts in the environment. Nimbus regulation therefore roughly corresponds to confidentiality and autonomy. Awareness, which is defined as a functional composition of focus and nimbus, is analogous to the dialectic negotiation of privacy boundaries.

This paper continues where Boyle and Greenberg left this discussion, trying to give formal semantics to such a conception of privacy and awareness. The model we introduce in this paper is based on Rodden's abstract version of the focus-nimbus model. We show how this model can provide a sound basis for describing mathematically the design space of awareness systems, in terms of the content exchanged, elementary user behaviors pertaining to sharing information about themselves or perceiving information about others. The sections that follow shall introduce the model and demonstrate how some principles for the protection of user privacy can be expressed succinctly, lending clarity and conciseness to the discussion of awareness systems and their design. 


\section{Model Overview}

Where the original focus/nimbus model describes how much aware two entities are about each-other in a particular space, our model describes what are the entities aware of regarding each-other in a particular situation. The model we propose is an extension of the focus/nimbus model, populated with the notions of entities, aspects, attributes, resources and observable items. These notions are introduced below with the help of the following scenario:

"John and Anna are seniors living alone; sometimes they invite each other for a walk. They like to do this easily and without social pressure on each other so they recently, installed a system that helps them convey their wish for a walk. When they feel like walking, they can flick a switch installed in their living room; the system indicates their intentions to the other side by lighting a small lamp in a visible position in the living room."

Entities are representations of actors, communities, and other agents (possibly artificial) within an awareness-system. The actors of the above scenario (i.e. John and Anna) are represented in an awareness system with the corresponding entities.

Aspects are any characteristics that refer to an entity's state. An aspect is actually the complement to the incomplete-statement "I want to be aware of your ...". In our scenario "John wants to be aware of Anna's wish for a walk"; thus the phrase "wish for a walk" is an aspect, i.e. a characteristic of Anna's state that may be shared with John. The notion of aspect is broad and loose enough encompassing more detailed terms like "location", "activity", "aspirations", or even "focus", and "nimbus".

Attributes are the place-holders in our model for the information exchanged between Entities. An attribute can be thought of as a potential answer to the request "Tell me something about your ' $X$ aspect'”. In our scenario an answer to John's request "Anna tell me something about your 'wish for walk'" could be "My 'wish for walk' is moderate"; thus the answer "My 'wish for walk' is moderate" is an attribute, binding the value "moderate" to the aspect "wish for walk".

In any situation an entity makes its state available to other entities using one or more attributes. Awareness though is dynamic. One's nimbus is populated with attribute-providers; i.e. functions that return those attributes that one makes available to other entities in a specific situation.

A resource is a binding of an aspect with a way of displaying one or more attributes about this aspect. In any situation an entity might employ one or more resources to express its interest about certain aspects of other entities. Roughly speaking a resource is a statement such as "I shall display the attributes you provide to me about your ... by ...". In our example, "John plans to display the attributes that Anna provides to him about her wish for walk by turning the lamp either on or off".

One's focus is populated with resource-providers; i.e. functions that return one's resources that are engaged to display information about other entities in a specific situation.

An observable item is the result of displaying some attributes about an aspect using a resource. Roughly speaking an observable item contains the answer to the question "How are these attributes displayed to you?". In our scenario a possible answer to the question "How is 'moderate wish for walk' displayed to you?' could be "by dimming the light on my desk". 
The negotiation of the reciprocal foci and nimbi of two entities in a given situation (i.e. the corresponding 'produced' attributes and resources) is a function which returns the observable-items that are displayed to the two entities about each other's states, effectively characterizing their reciprocal awareness.

In the above scenario, John indicates his wish to go for a walk to Anna using the walkswitch. We can consider that John's Nimbus contains an Attribute-Provider that returns(in any situation) an attribute about John's wish for walk based on the state of the walk-switch. On the other hand, Anna can check John's wish-for-walk by watching the corresponding lamp. System-wise we can consider that Anna's Focus is expressed via a resource that switches the lamp on/off depending on John's wish for walk. Needless to say that neither the walk-switch nor the lamp imply necessarily that Johns does actually wish to walk (he may forget to push the switch) or that Anna does notice the lamp (their respective actual/inherent nimbus and focus). However, we can imagine that Anna can unplug the lamp or even "assign" it to another person. So, Anna becomes aware of John's mood for walk, by manipulating her focus. Similarly, we can imagine that John could choose not to let Anna know about the state of the walk-switch, thus John lets Anna become aware of his mood for walk by manipulating his nimbus.

\section{Observable Items and Awareness}

"John is sitting on his sofa reading a magazine. Behind him, on his desk the walklamp illuminates indicating that his friend Anna feels like going for a walk.”

In this situation the illuminating-lamp is an Observable Item that indicates to John whether Anna wants to go for a walk. It should be stressed here that by the term observable we do not imply that John is seeing the lamp or even whether John perceives it as an indication for Anna's wish to go for a walk. We only stipulate that the lamp is available for observation, and that it is possible (in principle) for John to perceive. John's lamp may be switched-on whether he is looking at it or not. We should also stress that the term observable does not imply a modality; information may be presented in any perceivable manner (auditory, visual, tactile, etc..).

Taking the above example as a basis, we can assert that in any situation there is a set of observable items that a given entity can observe. In the context of an awareness system we can consider that an entity $i$ becomes aware about the state of entity $j$ through an awareness-characteristic function $a_{i j}$ which under a given situation $r$ returns the set of observable by entity $i$ items that present information regarding entity $j$ :

$$
\forall i, j: \text { Entity; } a_{i j}: \text { RealSituation } \rightarrow \mathbb{F} \text { ObservableItem; }
$$

In this section and elsewhere RealSituation is an abstraction that we use to encapsulate the dynamic nature of the universe to which awareness refers. The model itself is neutral regarding the notion of reality; the model and the user-related properties in the following sections do not make any assumptions about what is "real".

The exact semantics of $a_{i j}$ will be shaped out, as we advance in the notions of focus, and nimbus. For convenience, we use $a_{i j}^{r}$ to denote $a_{i j}(r)$.

As an example of an ObservableItem we can consider a function that returns an ObservableItem (light illumination):

$$
\text { lightIlumination: Lamp } \times \text { Voltage } \rightarrow \text { ObservableItem; }
$$


We will not define the function lightIllumination in detail but we can imagine that this function returns the effect of applying the specified voltage on a lamp source. For example lightIlumination(lamp1,240V) represents an observable item that originates from applying 240Volts on lamp1.

In the aforementioned scenario we can state that

$$
a_{\text {John, Anna }}^{r}=\{\text { lightIllumination }(\operatorname{lamp} 1,240 \mathrm{~V})\}
$$

i.e. the awareness of John about Anna in a situation $(r)$ is a set that includes one observable item that indicates Anna's wish to walk by illuminating lamp1. Note that it would be more appropriate to say "potential awareness", since we have no information about John's physical (inherent) focus. For brevity, we use instead the term "awareness" and we imply a corresponding interpretation for statements such as "John is aware of Anna's wish for a walk".

\section{Attributes, Attribute Providers and Nimbus}

Nimbus represents a sub-space across which an entity makes its state available to others. We can consider that in any real situation an entity's state(as it is presented to other entities) holds information about a wide range of aspects; we use the scheme "Attribute" to describe a piece of information( "value") about an aspect("aspect").

- Attribute

aspect : Aspect;

value : Data;

For convenience, we use the idiom $(a: v)$ to denote the attribute

$\langle$ aspect $\leadsto$ a,value $\sim v\rangle$, i.e. the attribute about aspect a with value $v$

There may be more than one attributes about the same aspect for a single entity; for example one's state may include an attribute about "location" with value "home"(location:home), and an other attribute also about "location" with the value "kitchen" (location: kitchen). Notice that the model does not preclude that one's state may include contradictory attributes (allowing for imperfect technology or intentional misinformation by the user).

One's attributes and the entities that they are available to may change over time. We define a function-type AttributeProvider, that when applied to a real situation returns an attribute and the set of entities that this attribute is made available to. Hence, an attribute provider may return different attributes available to different entities depending on the situation:

$$
\text { AttributeProvider }::=\text { RealSituation } \rightarrow(\text { Attribute } \times \mathbb{F} \text { Enitity })
$$

For an instance of AttributeProvider $p$ we use $p^{r}$ to denote first $p(r)$ and $p^{r}$.e to denote second $p(r)$; i.e. $p^{r}$ denotes the attribute that $p$ returns at situation $r$, and $p^{r} . e$ denotes the set of entities that $p^{r}$ is made available to. 
For each entity $i$ we assume that nimbus $s_{i}$ includes all the entity's $i$ attribute providers:

$$
\forall i: \text { Entity; } \text { nimbus }_{i}: \mathbb{F} \text { AttributeProvider }
$$

Given $\operatorname{nimbus}_{i}$, we can define a function $n_{i j}$ such that when applied to a real situation it returns the attributes of $i$ that are available to $j$ :

$$
\begin{gathered}
\forall r: \text { RealSituation; } i, j: \text { Entity; } n_{i j}: \text { RealSituation } \rightarrow \mathbb{F} \text { Attribute } \mid \\
n_{i j}(r)=\left\{a: \text { Attribute }\left(\exists p: \text { AttributeProvider } ; p \in \operatorname{nimbus}_{i} \bullet\left(a=p^{r}\right) \wedge\left(j \in p^{r} . e\right)\right)\right\}
\end{gathered}
$$

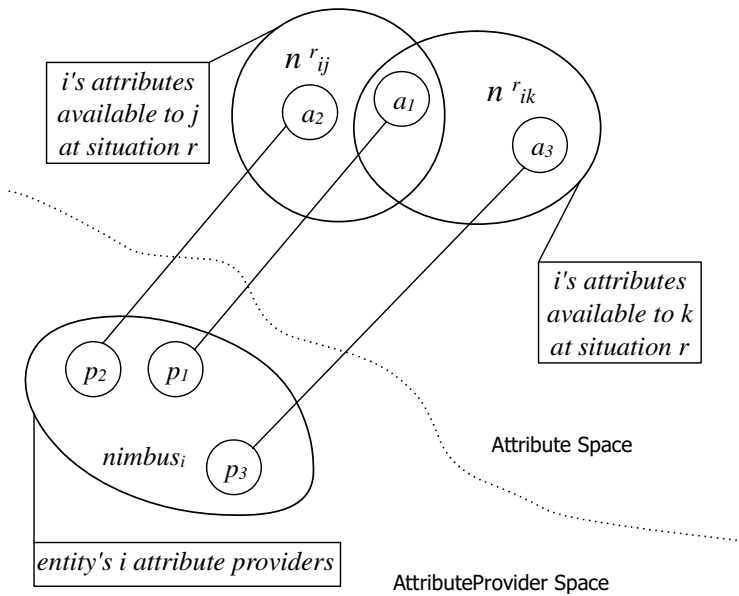

Fig. 1. The nimbus of entity $i$ to entities $j$ and $k$

Figure 1 shows three attribute providers of entity $i(p 1, p 2, p 3)$, and their corresponding attributes in a situation $r$ (i.e. $a 1, a 2, a 3$ ). Attribute provider $p 2$, makes attribute $a 2$ available to entity $\mathrm{j} ; p 1$ makes $a l$ available to entities $j$, and $k ; p 3$ makes $a 3$ available to entity $k$. Consequently the nimbus of entity $i$ to $j$ at this situation is $n_{i j}^{r}=\{a 1, a 2\}$ and the nimbus of entity $i$ to $k$ at this situation is $n_{i k}^{r}=\{a 1, a 3\}$.

Previously it was noted that the model does not preclude that one's state may include contradictory attributes. For example an attribute about location with value home (location: home), contradicts the attribute (location: away). We populate the attribute space with a relationship that denotes contradicting attributes:

$$
\forall a, b: \text { Attribute; a contradicting } b \Leftrightarrow(a, b) \in \text { _contradicting_; }
$$

It was also noted that there may be more than one attributes about the same aspect for a single entity. Furthermore, one may agree that an attribute(al) about aspect "activity" with a value "sleeping" implies an attribute $(a 2)$ about aspect "location" with a value "bed", and the latter may imply an attribute(a3) about "location" with value "home" and so on. The exact ontological relationships and whether an ontology can be global, or application specific, or entity specific, or moreover situation-specific is out of the context of this paper. However given an ontological relationship between attributes: 
We can define a function that returns all possible attributes that are implied from a single attribute:

$$
\begin{gathered}
\text { impliedAttributes : Attribute } \rightarrow \mathbb{F} \text { Attribute } \\
\forall \text { a:Attribute; impliedAttributes }(a)=\left\{\text { u:Attribute }(a, u) \in \text { implies_}_{-}^{*}\right\} \\
\text { where_implies_* is the reflexive transitive closure of _implies_ }
\end{gathered}
$$

More generally we can to take into account implications from attribute tuples, triads, quads, or from any set of attributes; we assume that the "impliedAttributes" function is extended to return all attributes implied from a set of attributes:

$$
\text { impliedAttributes : } \mathbb{F} \text { Attribute } \rightarrow \mathbb{F} \text { Attribute; }
$$

The exact definition of this extensive function is out of scope; given its existence however, we can define $n^{*}{ }_{x y}$ to return all implied attributes of $n^{r}{ }_{x y}$.

$$
\left.\forall r \text { :RealSituation; } n^{*}{ }_{i j}=\left\{a \text { :Attribute } \mid a \in \text { impliedAttributes }\left(n^{r}{ }_{i j}\right)\right\}\right\}
$$

\subsection{Nimbus Example}

We can reflect on the nimbi of John and Anna in the scenario introduced earlier; John lets Anna know if he feels like walking by turning the switch on/off. In terms of the system John makes available to Anna in any situation $r$, an attribute $a$ ( $\left.a \in n_{\text {John,Anna }}^{r}\right)$ about his "wishforWalk". John's nimbus contains an attribute provider that in any real situation returns the aforesaid attribute, and adjusts the attribute's value according to the state of the switch:

sw 1: AttributeProvider; sw $1 \in$ nimbus $_{\text {John }} \mid \forall r$ RealSituation; $\left(\right.$ sw $1^{r}$.aspect $=$ wishforWalk $) \wedge$

$\left(\right.$ sw $1^{r}$.value $=$ if switchclosed $($ switch $1, r)$ then true else false $) \wedge\left(\right.$ sw $1^{r} . e=\{$ Anna $\left.\}\right)$

Thus, $s w l$ is an attribute provider in John's nimbus, which when applied in a situation $r$ it returns an attribute $\left(s w l^{\mathrm{r}}\right.$.aspect: $s w l^{\mathrm{r}}$.value) and an entity set $\left(s w l^{\mathrm{r}} . e\right)$ that includes Anna. The attribute's aspect is wishforWalk and its value is either true or false (depending on the state of switchl).

Now we can wrap up John's nimbus $\left(\right.$ nimbus $\left._{\mathrm{John}}\right)$

$$
\operatorname{nimbus}_{J o h n}=\{s w 1\}
$$

Using the definition of $n_{i j}$ we can verify that:

$$
\forall r: \text { RealSituation; } n_{\text {John, John }}^{r}=\varnothing ; n_{\text {John, Anna }}^{r}=\left\{s w 1^{r}\right\} ;
$$

Similarly for Anna and her installation:

$$
\begin{array}{r}
\text { sw2: AttributeProvider; sw2 } \in \text { nimbus } s_{\text {Anna }} \mid \forall r: \text { RealSituation; } \\
\left(\text { sw } 2^{r} . \text { aspect }=\text { wishforWalk }\right) \wedge \\
\left(\text { sw } 2^{r} \text {.value }=\text { if } \begin{array}{l}
\text { switchclosed }(\text { switch } 2, r) \text { then true else false }) \wedge \\
\left(\operatorname{sw} 2^{r} . e=\{\text { John,Anna }\}\right)
\end{array}\right.
\end{array}
$$

Anna's nimbus will be

$$
\operatorname{nimbus}_{\text {Anna }}=\{s w 2\}
$$

Using the definition of $n_{i j}$ we can verify that:

$$
\forall r: \text { RealSituation; } n_{\text {Anna, John }}^{r}=\left\{s w 2^{r}\right\} ; n_{\text {Anna, Anna }}^{r}=\left\{s w 2^{r}\right\} ;
$$


Note that Anna's "wishForWalk" is available both to John and to herself, in contrast with John who makes available his "wishForWalk" only to Anna. This may sound awkward, however it points-out the fact that an entity is-not/can-not-be de facto aware of the information that is collected about it and made available to others (it might not be aware, e.g., in case of covert surveillance). Further this observation points out that nimbus does not imply a physical location or ownership of the underlying attribute providers.

\section{Resources, Resource-Providers and Focus}

Focus represents a sub-space within which an entity focuses its attention. Systemwise we assume that an entity has a limited set of resources to represent the provided attributes regarding aspects of other entities. The scheme Resource describes an aspect of interest and a function that transforms the corresponding attributes to an observable item.

- Resource

aspect : Aspect;

render : $\mathbb{F}$ Attribute $\rightarrow$ ObservableItem;

Note that an entity may assign more than one resources that render the same aspect(s) of another entity. E.g., John can render Anna's wishForWalk both by a lamp at home and an icon on his mobile phone.

One's resources may change depending on the situation; to incorporate this in the model we define a function-type ResourceProvider, that when applied to a real situation returns a resource and an entity that it is assigned to. Hence, a single resource provider may return different resources assigned to different entities depending on the situation:

$$
\text { ResourceProvider }::=\text { RealSituation } \rightarrow(\text { Resource } \times \text { Entity })
$$

For a ResourceProvider instance $p$ we use $p^{r}$ to denote first $p(r)$ and $p^{r}$.e to denote second $p(r)$. Hence $p^{r}$ denotes the resource that $p$ returns at the situation $r$, and $p^{r} . e$ denotes the entity that $p^{r}$ is assigned to. For each entity we assume that focus includes the set of entity's $i$ resource providers.

$$
\forall i: \text { Entity }_{\text {focus }} \text { : } \mathbb{F} \text { ResourceProvider }
$$

Given focus $_{i}$ we define $f_{i j}$ to return only those resources of $i$ that focus on entity $j$ :

$$
\begin{aligned}
\forall r: \text { RealSituation } ; \forall i, j: \text { Entity } ; f_{i j}: \text { RealSituation } \rightarrow \mathbb{F} \text { Resource } \mid \\
f_{i j}(r)=\left\{c: \text { Resource } \mid\left(\exists p: \text { ResourceProvider } ; p \in \text { focus }_{i} \bullet\left(c=p^{r}\right) \wedge\left(j=p^{r} . e\right)\right)\right\}
\end{aligned}
$$

In figure 2 we can notice on the bottom left three resource providers of entity's $i$ focus (i.e. $p 1$ p 2 p3), and their corresponding resources in a situation $r$ (i.e. $r 1, r 2, r 3$ ). The resource provider $p 1$, assigns the resource $r l$ to display information from entity $j$; $p 2$ assigns $r 2$ to $j$; $p 3$ assigns $r 3$ to $k$. Consequently the focus of entity $i$ on $j$ at this situation is $f^{r}{ }_{i j}=\{r 1, r 2\}$ and the focus of entity $i$ on $k$ at this situation is $f^{r}{ }_{i k}=\{r 3\}$. 


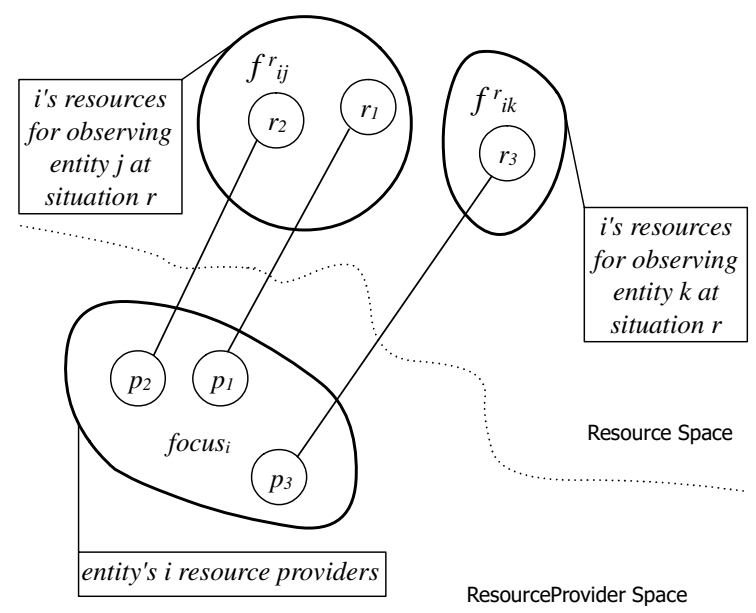

Fig. 2. Focus of entity i upon entities $\mathrm{j}$ and $\mathrm{k}$

\subsection{Focus Example}

Continuing our example, imagine that "John uses a lamp to display Anna's wish for a walk and vice versa". A lamp (resource) is assigned to display Anna's wishForWalk. System wise, John's focus on Anna contains a resource $r\left(r \in f^{r}{ }_{\text {John,Anna }}\right)$ that renders attributes about the aspect “wishforWalk". John's focus ( focus $_{J o h n}$ ) contains a resource provider, that returns this resource and adjusts the resource's rendering (illumination) according to the attributes that the system provides:

$$
\begin{aligned}
& \text { wr 1: ResourceProvider; wr } 1 \in \text { focus }_{\text {john }} \mid \forall r \text { :RealSituation; } \\
& \left(\text { wr } 1^{r} \text {.aspect }=\text { wishForWalk }\right) \wedge \\
& \left(\forall s: \mathbb{F} \text { Attribute; wr } 1^{r} \text {.render }(s)=\right. \\
& \text { if }(\exists p: \text { Attribute } ; p \in s \mid \text { p.aspect }=\text { wishForWalk } \wedge \text { p.value }=\text { true }) \text { then } \\
& \text { lightIllumination(lamp 1,240V) else lightIllumination(lamp 1,0V)) } \wedge \\
& \text { (wr } 1^{r} . e=\text { Anna ) }
\end{aligned}
$$

Thus wrl is a ResourceProvider that returns a resource which renders attributes about wishforWalk either by turning on lampl or by turning it off; wrl.e denotes that the returned resource should be assigned to Anna. Consequently, wrl is a resource provider in John's focus, that when applied to a real situation $r$, it returns a resource that can render Anna's wishforWalk.

We can wrap up John's focus $\left(\right.$ focus $\left._{J o h n}\right)$ :

$$
\text { focus }_{J o h n}=\{w r 1\}
$$

We can apply the definition of $f_{i j}$ to verify:

$$
\forall r: \text { RealSituation; } f_{\text {John, John }}^{r}=\varnothing ; f_{\text {John, Anna }}^{r}=\left\{w r 1^{r}\right\} ;
$$

Similarly we can describe Anna's focus on John's wish for walk.

wr 2: ResourceProvider; $w r 2 \in$ focus $_{\text {Anna }} \mid \forall r$ :RealSituation;

$\left(w r 2^{r}\right.$.aspect $=$ wishForWalk $) \wedge$ 
$\left(\forall s: \mathbb{F}\right.$ Attribute; wr $2^{r}$.render $(s)=$

if $\left(\exists p:\right.$ Attribute; $p \in S \mid$ p.aspect $=$ wishForWalk ${ }^{\wedge}$ p.value $=$ true $)$ then lightIllumination(lamp2,240V) else lightIllumination(lamp2,0V)) $\wedge$ $\left(w r 2^{r} . e=J o h n\right)$

Consequently Anna's focus will be:

$$
\text { focus }_{\text {Anna }}=\{w r 2\}
$$

We can apply the definition of $f_{i j}$ to verify:

$$
\forall r: \text { RealSituation; } f_{\text {Anna, Anna }}^{r}=\varnothing ; f_{\text {Anna, John }}^{r}=\left\{w r 2^{r}\right\} ;
$$

\section{Focus/Nimbus Negotiation and Awareness-Systems}

Figure 3 shows the attributes that an entity " $j$ " makes available to an entity " $i$ " at a situation " $r$ " (i.e. $a 1, a 2, a 3)$ through $n_{j i}^{r}$. On the top-left we see their projection $(A)$ on the Aspect Space i.e. the aspects they refer to. For example the attribute $a_{1}$ contains information about aspect $Y$, so its projection on the aspect space is $Y$. We notice also the resources that $i$ assigns for observing $j$ at $r$ (i.e. $r 1, r 2)$ through $f^{r}{ }_{i j}$ and the resource projection $(B)$ on the Aspect Space; i.e. the aspects that the resources claim to (i.e. are set to) render. For example, the resource $r_{2}$ claims to render the aspect $X$, so its projection on the aspect space is $X$. The intersection $A \cap B$, represents the aspects that $i$ wants to observe about $j$, and $j$ is making available to $i$ at the situation $r$. Consequently, the set of items that $i$ can observe about $j\left(a_{i j}^{r}\right)$, are the result of rendering those attributes of $n_{i j}^{r}$ that project on $A \cap B$ (i.e. a2, and $a 3$ ), using those corresponding resources of $f^{r}{ }_{i j}$ that project on $A \cap B$ (i.e. $r 1$ ); therefore (see bottom of figure 3 ) $a_{i j}^{r}$ includes the observable item $o 1=r 2$.render $(\{a 2, a 3\})$.

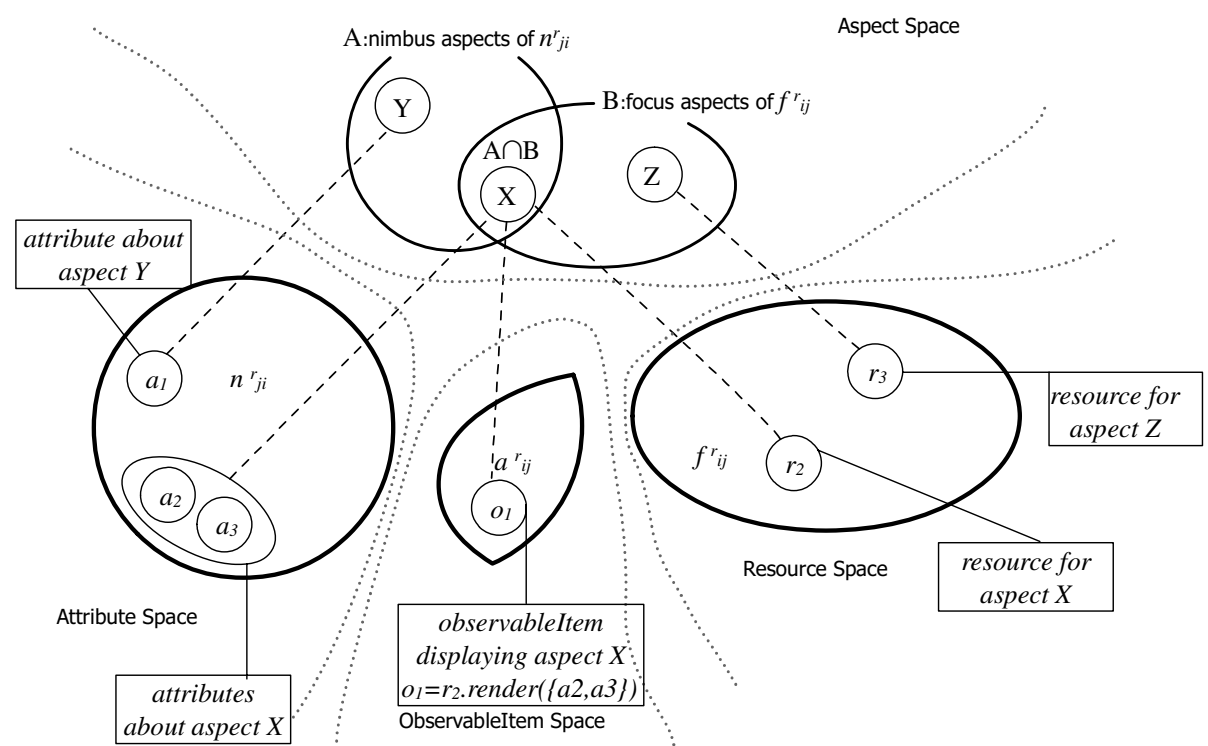

Fig. 3. Illustration of focus/nimbus negotiation and awareness that entity $i$ has of entity $j$ 
We generalize this negotiation of the reciprocal foci, and nimbi between two entities as follows:

$$
\begin{gathered}
a_{i j}::=\text { RealSituation } \rightarrow \mathbb{F} \text { ObservableItem; } \\
\forall r: \text { RealSituation } ; \\
a_{i j}(r)=\left\{v: \text { ObservableItem } \mid\left(\forall c: \text { Resource } ; c \in f^{r}{ }_{i j}^{\bullet}\right.\right. \\
\left.v=\text { c.render }\left(\left\{u: \text { Attribute } \mid\left(u \in n_{j i}^{r}\right) \wedge(\text { u.aspect }=\text { c.aspect })\right\}\right)\right\}
\end{gathered}
$$

Returning to our example, John's observable items about Anna's state is the result of rendering the value of Anna's wishforWalk as it is provided to John (i.e. $s w 2^{r}$ ) using the resource that John assigned for this purpose (i.e. $w r l^{r}$ ). Conversely, Anna's observable items about John's state is the result of rendering the value of John's wishforWalk as it is provided to Anna (i.e. $s w l^{r}$ ) using the resource that Anna assigned for this purpose(i.e. $w r 2^{r}$ ). On the other hand both $a_{\text {John,John }}^{r}$, and $a_{\text {Anna,Anna }}^{r}$ are empty sets, since John's nimbus to himself is an empty set, and in the case of Anna, although her wishforWalk is available to her-self, there is no resource assigned to render it:

$$
\begin{gathered}
\forall r: \text { RealSituation; } \\
a_{\text {John,Anna }}^{r}=\left\{w r 1^{r} \cdot \text { render }\left(\left\{s w 2^{r}\right\}\right)\right\} ; a_{\text {John,John }}^{r}=\varnothing ; \\
a_{\text {Anna, John }}^{r}=\left\{w r 2^{r} \cdot \text { render }\left(\left\{s w 1^{r}\right\}\right)\right\} ; a_{\text {Anna,Anna }}^{r}=\varnothing ;
\end{gathered}
$$

At this point we can wrap together the definitions so far in a scheme that describes an awareness system. The scheme defines the set of entities in a system, their nimbi and foci, as well as their reciprocal awareness information using the definitions we have introduced so far:

$\left[\begin{array}{ll}\text { Awareness System } & \\ \text { enitities } \quad: \mathbb{F} \text { Entity } ; \\ \text { nimbus } \quad: \text { Entity } \rightarrow \mathbb{F} \text { AttributeProvider } ; \\ \text { focus } \quad: \text { Entity } \rightarrow \mathbb{F} \text { ResourceProvider } ; \\ n:(\text { Entitiy } \times \text { Entity }) \rightarrow(\text { RealSituation } \rightarrow \mathbb{F} \text { Attribute }) ; \\ f:(\text { Entitiy } \times \text { Entity }) \rightarrow(\text { RealSituation } \rightarrow \mathbb{F} \text { Resource }) ; \\ a:(\text { Entitiy } \times \text { Entity }) \rightarrow(\text { RealSituation } \rightarrow \mathbb{F} \text { ObservableItem }) ;\end{array}\right.$

$$
\begin{aligned}
& \text { dom nimbus=entities; dom focus =entities; } \\
& \forall r: \text { RealSituation; } i, j: \text { Entity; } i, j \in \text { entities; } \\
& \forall u: \text { Attribute; } c \text { : Resource; } v: \text { ObservableItem } \\
& r \mapsto u \in n_{i j} \Leftrightarrow \exists p: \text { AttributeProvider; } p \in \text { nimbus }_{i} \mid\left(u=p^{r}\right) \wedge(j \in \text { u.access }) \\
& \left.r \mapsto c \in f_{i j} \Leftrightarrow \exists p: \text { ResourceProvider; } p \in \text { focus }_{i} \mid c=p^{r}\right) \wedge(j=\text { c.entity }) \\
& r \mapsto v \in a_{i j} \Leftrightarrow \exists p: \text { Resource; } p \in f^{r} \mid \\
& \left.\left.\quad v=p . r e n d e r\left(\left\{u: \text { Attribute } \mid\left(u \in n^{r}{ }_{j i}\right) \wedge(\text { u.aspect=c.aspect })\right\}\right)\right)\right\}
\end{aligned}
$$

We use the idioms nimbus for $_{i}$ nimbus(i), focus $s_{i}$ for focus $(i), n_{i j}$ for $n(i, j), f_{i j}$ for $f(i), a_{i j}$ for $a(i, j), n_{i j}^{r}$ for $n(i, j)(r), f^{r}{ }_{i j}$ for $f(i, j)(r), a_{i j}^{r}$ for $a(i, j)(r)$.

In the following sections we will demonstrate how the introduced model allows us to reason about some privacy related properties of awareness systems. 


\section{Plausible Deniability}

The term plausible deniability has been often used (e.g., see [3],[1]) to describe how users of communication systems may rely on ambiguity in order to have a plausible excuse for avoiding communication or interaction with a third party.

Price et al. [3] explore the social need for plausible deniability in ubicomp systems and in relation with one's location and identity. As they point out, many systems depart from social norms that are otherwise present in face-to-face interactions (where a person can easily see whether he/she is being observed by others). Price et al classify five types of user controlled "noise" to protect location privacy (Anonymizing, Hashing, Cloaking, Blurring, and Lying).

In a similar line, Lederer et al. [16] report that people decide to disclose information about their activities and location based on the identity of the requester and the situation in which it happens. Consolvo et al. [7] introduce several requirements for location-aware applications. Among these they mention the need to support denial (e.g. the ability not to disclose any information), and deception (e.g. the ability to deceive in the response). In their studies, blurring (i.e. the ability to disclose less specific information) was encountered less frequently. Summarizing, we can identify three basic deceptive patterns:

- Deception/Lying: intentionally false information

- Denial/Cloaking: no information disclosure

- Blurring/Evasion: revealing part of the information

These are discussed below in terms of the model of awareness introduced.

\subsection{Deception / Lying}

Lying can be thought as giving intentionally false information about an aspect. We consider that an entity is lying when it is giving to some other entity contradicting information compared to itself about an aspect.

For example, consider an entity " $a$ " that makes available to itself an attribute (location: home) whereas it makes available to entity " $b$ " an attribute (location: away). Given that (location: home) is contradictory to (location: away) we can state that " $a$ " is lying to " $b$ " about its location.

Bearing in mind a simple ontology like the one we described earlier, if entity " $a$ " would make (activity:sleeping) available to it-self then the predicate "a is lying to $b$ about its location" would still hold since in the context of the specific ontology, the attribute (activity: sleeping) implies (location: home) which contradicts to the attribute (location: away). Following the above we can formalize deception/lying:

$$
\begin{aligned}
& \text { _isLyingTo_About_: RealSituation } \rightarrow \mathbb{P}(\text { Entity } \times \text { Entity } \times \text { Aspect }) \\
& \forall r \text { :RealSituation; } x, y \text { Entity; a:aspect; } \\
& x \text { isLyingTo y About } a(r) \Leftrightarrow(x, y, a) \in \text { _isLyingTo_About_ }(r) \Leftrightarrow \\
& \exists u, v \text { :Attribute } \mid u \in n^{{ }^{*}{ }_{x y}} \wedge v \in n^{{ }_{r}}{ }_{x x} \wedge \text { u.aspect }=a \wedge u \text { contradicting } v
\end{aligned}
$$

i.e., $x$ is lying to $y$ about an aspect $a$, when there is at least one attribute about $a$ that is made available to $y$ (explicitly or by implication), such that it contradicts with an attribute that $x$ makes available to him/her-self (explicitly or by implication). 


\subsection{Denial / Cloaking}

Price describes "Cloaking" as the ability to hide one's location or identity. More generally, cloaking can concern any aspect of one's nimbus. Hence we consider cloaking as the ability to conceal any attributes about an aspect of an entity from another entity.

For example, consider an entity " $a$ " that makes no attributes available to entity " $b$ " about its location, where as it makes available to an entity " $c$ " an attribute (location: home). We can say in this example that $a$ is hiding its location from $b$.

Taking in account a simple ontology like the one described earlier, we could say that even if only an attribute (activity:sleeping) would be available to entity " $c$ " the predicate " $a$ is hiding its location from $b$ " would still hold since in the context of the specific ontology, (activity: sleeping) implies several attributes about location such as (location: bedroom) and (location: home). Therefore in the formal definition that follows we use $n^{*}{ }_{x y}$ which actually contains all the possible implied attributes of $n_{x y}^{r}$.

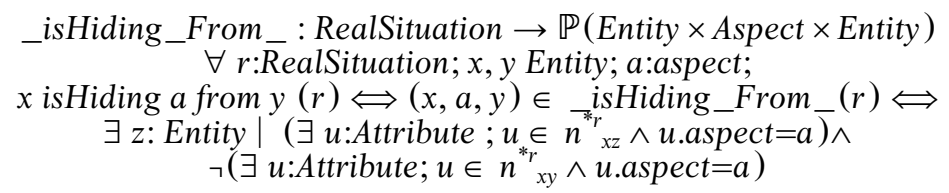

i.e., $x$ is hiding an aspect $a$ from $y$, when there are no attributes about $a$ that are made available to $y$ either explicitly or by implication, and at the same time there is at least one attribute about $a$ that $x$ makes available to an other entity $z$. Note that $z$ can be any entity including $x$ it-self.

\subsection{Blurring / Evasion}

In contrast with Cloaking, Blurring is not hiding an aspect, but rather it concerns withholding information. Price describes "blurring" as the ability to decrease the precision of one's location. In a wider context we can replace "location" with any aspect of one's nimbus. To account with the term "decrease" we define "blurring" in comparison to a reference entity. Hence we consider that an entity is blurring information about an aspect to another entity, when the first is revealing less information about this aspect to the latter than a reference entity.

Before proceeding to a formal definition let's consider the phrase "less information about an aspect". This phrase implies that we need to take in account the term "information about an aspect". For that, we introduce a function attributesAbout, that when applied on a set of attributes and an aspect, it returns only those attributes that concern the specified aspect:

$$
\begin{gathered}
\text { attributesAbout }: \mathbb{F} \text { Attribute } \times \text { Aspect } \rightarrow \mathbb{F} \text { Attribute } \\
\forall s: \mathbb{F} \text { Attribute; a: Aspect; attributesAbout }(s, a)=\{u: \text { Attribute } ; u \in s \mid \text { u.aspect }=a\}
\end{gathered}
$$

To evaluate the expression "less information" we consider that if an attribute-set $s$ is a subset of an attribute-set $t$, then the set $s$ contains less information than the set $t$. For example a set that includes an attribute about location with value home (location: home) contains less information than the set \{(location: home), (location: bedroom)\} since the first set a subset of the latter. 
Taking in account a simple ontology like the one described earlier, we can tell that the set \{(location: home)\} contains less information than the set \{location: bedroom $)\}$, since the latter implies the first. Moreover $\{($ location:home $)\}$ contains less information than the set \{(activity: sleeping)\} since the latter implies both (location: bedroom) and (location: home). Consequently in the formal definition that follows we use $n^{{ }^{*} r}$ which actually contains all the possible implied attributes of $n_{x y}^{r}$.

$$
\begin{aligned}
& \text { _isBlurring_to_ }: \text { RealSituation } \rightarrow \mathbb{P}(\text { Entity } \times \text { Aspect } \times \text { Entity }) \\
& \text { let } x, y \text { : Entity; } a \text { :Aspect; } r \text { : RealSituation; } \\
& x \text { isBlurring a to } y(r) \Leftrightarrow(x, a, y) \in{ }_{*_{r}} \text { isBlurring_to_ }(r)_{*_{r}} \Leftrightarrow \\
& \exists z \text { : Entity | attributesAbout }\left(n ^ { { } ^ { * } { } _ { x y } , a ) } \overline { \subset } \text { attributes } \overline { A } \text { bout } \left(n^{\left.{ }^{*}{ }_{x z}, a\right)}\right.\right.
\end{aligned}
$$

i.e. $x$ is blurring information about an aspect $a$ to $y$, when all the attributes about $a$ that are made available to $y$ (explicitly or by implication), are a subset of the attributes about $a$ that are made available to an entity $z$ (explicitly or by implication). Note that the reference entity $z$ can be any entity including $x$ itself.

\section{Discussion on Physical/Inherent Awareness}

So far we have considered observable items without taking into account whether physical entities (such as actors) are indeed physically (inherently) aware of them. This is a point where one can utilize the quantitative notion of modeling awareness with Rodden's focus/nimbus model. We can actually consider that each observableItem has an inherent/physical nimbus, and each entity has an inherent focus. The composition of an entity's inherent focus with an observable item's inherent nimbus defines how aware an actor is of the observable item it self. If we assume that a system has sufficient resources/capabilities to apply Rodden's focus-nimbus model in the EntityObservableItem relationship (i.e. we can define the focus/nimbus composition), then we can reason in detail about the information (observable-items) that one is aware of.

For that we may consider a function $n^{+}$that associates an ObservalbeItem with its inherent nimbus in any situation, a function $f^{+}$that associates an Entity with its inherent focus in any situation, and an awareness quantifier function $a^{+}$:

$$
\begin{gathered}
n^{+}: \text {RealSituation } \times \text { ObservableItem } \rightarrow \text { InherentNimbus; } \\
f^{+}: \text {RealSituation } \times \text { Entity } \rightarrow \text { InherentFocus; } \\
a^{+} \text {InherentFocus } \times \text { InherentNimbus } \rightarrow \text { InherentAwareness }
\end{gathered}
$$

For an entity $x$, and an observableItem $u, a^{+}\left(f^{+}(r, x), n^{+}(r, u)\right)$ quantifies the question "How aware is entity $x$ of observable item $u$ at situation $r$ ". Using a predefined threshold $h$ we can state that $x$ is aware of $u$ at situation $r$ when its inherent awareness $a^{+}\left(f^{+}(r, x), n^{+}(r, u)\right)$ is greater than the predefined threshold:

$$
\begin{gathered}
\text { _isPhysicallyAwareOf_: RealSituation } \rightarrow(\text { Entity } \times \text { ObservableItem }) \bullet \\
\forall x: \text { Entity; } u: \text { ObservableItem; } r: \text { RealSituation; } \\
x \text { isPhysicallyAwareOf } u(r) \Leftrightarrow(x, u) \in \_i s \text { PhysicallyAwareOf_}(r) \Leftrightarrow \\
a^{+}\left(f^{+}(r, x), n^{+}(r, u)\right)>h
\end{gathered}
$$

Now we can define intentionally/unintentionally perceived awareness information; we can consider that entity $x$ is intentionally aware of an observable item $u$ when an $x$ is aware of $u$, and $u$ is one of the items that are generated through the system for that entity: 


\section{_isIntentionallyAwareOf_: RealSituation $\rightarrow($ Entity $\times$ ObservableItem $) \bullet$ $\forall r$ :RealSituation; $x:$ Entity; $u:$ ObservableItem • \\ $x$ isIntentionallyAwareOf $u(r) \Leftrightarrow(x, u) \in$ isIntentionallyAwareOf_ $(r) \Leftrightarrow$ ( $x$ isPhysicallyAwareOf $u(r)) \wedge\left(\exists y:\right.$ Entity $\left.\mid u \in a^{r}{ }_{x y}\right)$}

Similarly we can consider that entity $x$ is unintentionally aware of an observable item $u$ when an $x$ is aware of $u$, but $u$ is not anyone of the items that are generated through the system for that entity:

$$
\begin{gathered}
\quad \text { isUnintentionallyAwareOf_: RealSituation } \rightarrow(\text { Entity } \times \text { ObservableItem }) \\
\forall r: \text { RealSituation; } x: \text { Entity; } u: \text { ObservableItem } \bullet \\
x \text { isUnintentionallyAwareOf } u(r) \Leftrightarrow(x, u) \in \in_{-} \text {isUnintentionallyAwareOf_}(r) \Leftrightarrow \\
(x \text { isPhysicallyAwareOf } u(r)) \wedge \neg\left(\exists y: \text { Entity } \mid u \in a_{x y}\right)
\end{gathered}
$$

One may doubt the feasibility of computing functions $n^{+}, f^{+}$, and $a^{+}$as they refer essentially to cognitive phenomena. Yet, one's focus may be approximated with varying degrees of success by knowing whether they are present in front of the computer, or even further, monitoring their head pose or even their eye-gaze. In other words, an entity's nimbus can approximate its inherent focus allowing reasonable approximations of $n^{+}, f^{+}$, and $a^{+}$. In our scenario a weight-sensor on a chair facing the lamp could be included in John's nimbus for some reason(e.g. to notify Anna about John's presence). Whether John is aware of the lamp is more likely when he sits on the chair, although not certain (he might have his eyes closed or be day-dreaming).

Although we can define a relationship that relates observable-items with the attribute(s) that they present successfully, we can not assume that if an entity is physically aware of an observable item, that the entity is also physically aware of the presented attribute(s), since we do not model the cognitive processes of awareness (e.g., the lamp can display Anna's wish-for-walk, John can be physically aware of the lamp, but still John at the same time may be unaware of Anna's wish-for-walk). Modeling user perception is outside the scope of the model presented here; such issues have been addressed by cognitive models elsewhere such as the model of unawareness[18].

\section{Conclusion}

We have introduced a formal model of awareness systems, based on the focus/nimbus model of Benford [2] and Rodden [19]. Where the original focus and nimbus model describes how much aware is entity $i$ of entity $j$ in a particular space our model describes what is entity $i$ aware of regarding entity $j$, in a particular situation.

We have demonstrated that the model allows the formal expression of abstract concepts such as focus, nimbus, awareness but also socially oriented behaviors such as blurring information about oneself, lying etc. The model presented here abstracts away from modeling the propagation of awareness information as in [22] and [11], or information flow modeling as in[5]. It advances the focus/nimbus model of [2],[19] in that it is explicit about the object of awareness: i.e. the relationship of the information an entity can potentially provide about itself to that actually observed by another entity. This is necessary for modeling the social aspects of awareness systems as shown above.

Currently we are extending this work to model related concepts such as social translucence, community awareness, intentionality and symmetry of awareness systems. In the next steps of our research, an end-user programming platform for 
awareness systems will be created where users will be allowed to easily tailor the behavior of their system to effect blurring, anonymity, symmetry etc. The model presented can guide the design of this experimental platform and provides the conceptual foundations for defining an ontology by which awareness information can be described and reasoned about.

\section{References}

1. Aoki, P., Woodruff, A.: Making Space for Stories: Ambiguity in the Design of Personal Communication Systems. In: Proc. CHI 2005, pp. 181-190 (2005)

2. Benford, S., Bullock, A., Cook, N., Harvey, P., Ingram, R., Lee, O.: From rooms to cyberspace: models of interaction in large virtual computer spaces. Interacting with Computers 5(2), 217-237 (1993)

3. Price, B.A., Adam, K., Nuseibeh, B.: Keeping ubiquitous computing to yourself: A practical model for user control of privacy. International Journal of Human-Computer Studies 63(1-2), 228-253 (2005)

4. Boyle, M., Greenberg, S.: The Language of Privacy: Learning from Video Media Space Analysis and Design. ACM ToCHI 12(2), 328-370 (2005)

5. Bryans, J.W., Fitzgerald, J.S., Jones, C.B., Mozolevsky, I.: Formal Modelling of Dynamic Coalitions, with an Application in Chemical Engineering. In: Proc. of the 2nd Internaltional Symposium on Leveraging Applications of Formal Models (to appear)

6. Consolvo, S., Roessler, P., Shelton, B.E.: The careNet display: Lessons learned from an in home evaluation of an ambient display. In: Davies, N., Mynatt, E.D., Siio, I. (eds.) UbiComp 2004. LNCS, vol. 3205, pp. 22-29. Springer, Heidelberg (2004)

7. Consolvo, S., Smith, I., Matthews, T., LaMarca, A., Tabert, J., Powledge, P.: Location Disclosure to Social Relations: Why, When, \& What People Want to Share. In: Proc. of the Conference on Human Factors and Computing Systems: CHI 2005, pp. $81-90$ (2005)

8. Dourish, P., Belloti, V.: Awareness and Coordination in Shared Workspaces. In: Proceedings, CHI 1992, pp. 117-124. ACM Press, New York (1992)

9. Erickson, T., Halverson, C., Kellogg, W.A., Laff, M., Wolf, T.: Social translucence: designing social infrastructures that make collective activity visible. Commun. ACM 45(4), 40-44 (2002)

10. Fernado, O., Adach, K., Cohen, M.: Phantom Sources for Separation of Listening and Viewing Positions of Multipresent Avatars in Narrowcasting Collaborative Virtual Environments. In: Proceedings, ICDCSW 2004 (2004)

11. Fuchs, L., Pankoke-Babatz, U., Prinz, W.: Supporting cooperative awareness with local event mechanisms: The GroupDesk system. In: Proceedings of ECSCW 1995, pp. 247 262 (1995)

12. Hindus, D., Mainwaring, S.D., Leduc, N., Hagström, A.E., Bayley, O.: Casablanca: Designing social communication devices for the home. In: Proceedings CHI 2001, pp. 325$332(2001)$

13. Hong, J.I., Landay, J.A.: An Architecture for Privacy- Sensitive Ubiquitous Computing. In: Mobisys 2004, Boston, MA, pp. 177-189 (2004)

14. Iachello, G., Smith, I., Consolvo, S., Chen, M., Abowd, G.: Developing Privacy Guidelines for Social Location Disclosure Applications and Services. In: Proceedings of the Symposium on Usable Privacy and Security (SOUPS 2005) (2005)

15. Gao-feng, J., Yong, T., Yun-cheng, J.: A service-oriented group awareness model and its implementation. In: Lang, J., Lin, F., Wang, J. (eds.) KSEM 2006. LNCS, vol. 4092, pp. 139-150. Springer, Heidelberg (2006) 
16. Lederer, S., Mankoff, J., Dey, A.K.: Who Wants to Know What When? Privacy Preference Determinants in Ubiquitous Computing. In: Proceedings of Extended Abstracts of CHI 2003, ACM Conference on Human Factors in Computing Systems, Fort Lauderdale, FL, pp. 724-725 (2003)

17. Markopoulos, P., Romero, N., van Baren, J., IJsselsteijn, W., de Ruyter, B., Farshchian, B.: Keeping in touch with the family: home and away with the ASTRA awareness system. In: CHI Extended Abstracts 2004, pp. 1351-1354 (2004)

18. Modica, S., Rustichini, A.: Awareness and Partitional Information Structures. Theory Dec. 37, 107-124 (1994)

19. Rodden, T.: Populating the Application: A Model of Awareness for Cooperative Applications. In: Proc. ACM 1996 (CSCW 1996), pp. 87-96 (1996)

20. Rowan, J., Mynatt, E.D.: Digital family portrait field trial: Support for aging in place. In: Proc. CHI 2005, pp. 521-530 (2005)

21. Schmidt, K.: The Problem with Awareness Introductory Remarks on Awareness in CSCW. Computer Supported Collaborative Work 11(34), 285-298 (2002)

22. Simone, C., Bandini, S.: Integrating Awareness in Cooperative Applications through the Reaction-Diffusion Metaphor. Computer Supported Cooperative Work: The Journal of Collaborative Computing 11(3-4), 495-530 (2002)

\section{Questions}

\section{Michael Harrison:}

Question: Had you thought about using non-standard logics such as knowledge logics to express the information you're trying to express? See for example: Fagin, R., Halpern, J. Y., Moses, Y. and Vardi, M Y. Reasoning about knowledge,. MIT Press, Cambridge, Massachusetts, 1995.

Answer: We don't try to express knowledge as cognition. We haven't looked in that direction. Thank you for the reference.

\section{Fabio Paterno':}

Question: For what applications is the modeling appropriate?

Answer: For investigating how people can configure their awareness systems themselves. It helps to identify patterns within an awareness system. It allows people to configure awareness of their activities and supports lightweight communication systems.

\section{Morten Borup Harning:}

Question: Is the idea that your model can help modeling for privacy by making the awareness properties and interdependencies clearer?

Answer: Privacy is a concern of the model. We can describe aspects of behavior that are relevant to privacy.

\section{Anke Dittmar:}

Question: I can imagine that people would change their behavior if they make information explicit through an awareness system based on formalized descriptions of lying etc. and their knowledge about the system. Did you consider this in your formalization?

Answer: We have tried to keep as close as possible to what people actually do, but we haven't considered this particular issue so far. 